\title{
Antihypertensive Effects of Sesamin in Humans
}

\author{
Takashi MIYAWAKI ${ }^{1,2}$, Hideshi AONO ${ }^{2}$, Yoshiko TOYODA-ONO ${ }^{3}$, Hirofumi MAEDA ${ }^{3}$, \\ Yoshinobu KISO ${ }^{3}$ and Kenji MORIYAMA ${ }^{4}$ \\ ${ }^{1}$ NTT West Kansai Health Administration Center, 604, Bano-cho, Nakagyo-ku, Kyoto 604-8172, Japan \\ ${ }^{2}$ NTT West Kyoto Hospital, Kyoto 601-8441, Japan \\ ${ }^{3}$ Institute for Health Care Science, Suntory Ltd., Mishima-gun, Osaka 618-8503, Japan \\ ${ }^{4}$ School of Pharmaceutical Sciences, Mukogawa Women's University, Nishinomiya, Hyogo 663-8179, Japan \\ (Received July 25, 2008)
}

\begin{abstract}
Summary Sesamin, one of the lignans contained in sesame, has been considered to have medicinal effects. It has been reported that sesamin suppressed the development of hypertension in rats. In this study, using a double-blind, cross-over, placebo-controlled trial, we investigated the effect of 4-wk administration of sesamin on blood pressure (BP) in mildly hypertensive humans. Twenty-five middle-aged subjects with mild hypertension were divided into two groups, matched by age and body mass index. Twelve subjects were allocated to 4-wk intake of capsules with $60 \mathrm{mg}$ sesamin per day and 13 subjects to 4-wk intake of a placebo (period 1). After a 4-wk washout period, the subjects received the alternative administration for $4 \mathrm{wk}$ (period 2). BP decreased with statistical significance with the administration of sesamin (systolic: $137.6 \pm 2.2$ to $134.1 \pm 1.7 \mathrm{mmHg}, p=0.044$, diastolic: $87.7 \pm 1.3$ to $85.8 \pm 1.0 \mathrm{mmHg}, p=0.045$ ), but little changed with the placebo (systolic: $135.0 \pm 1.8$ to $135.1 \pm 1.7 \mathrm{mmHg}$, diastolic: $85.9 \pm 1.2$ to $86.6 \pm 1.2 \mathrm{mmHg}$ ). In conclusion, 4-wk administration of $60 \mathrm{mg}$ sesamin significantly decreased BP by an average of $3.5 \mathrm{mmHg}$ systolic BP and $1.9 \mathrm{mmHg}$ diastolic BP. These results suggest that sesamin has an antihypertensive effect in humans. Epidemiological studies suggested that a $2-3 \mathrm{mmHg}$ decrease in $\mathrm{BP}$ reduces the rate of cardiovascular diseases; therefore, it is considered that BP reduction achieved by sesamin may be meaningful to prevent cardiovascular diseases.
\end{abstract}

Key Words sesamin, hypertension, double-blind, cross-over study, antihypertensive effects

Sesame seeds have been commonly used as a traditional health food since ancient times in Asian regions. Sesamin seeds contain not only oil and protein but also characteristic lignans, such as sesamin (0.01-1.0\%) and sesamolin. Sesamin is epimerized during acid-clay bleaching in the oil refining process to form episesamin (Fig. 1) (1).

It seems that sesamin was absorbed via the portal vein in the native form and metabolized to the mono- or di-catechol compound by enzymes in hepatocytes. Both metabolites had antioxidant activity in the liver and were finally conjugated with glucuronic acid by glucuronidase for excretion into bile (2).

Several studies have shown the biological activities of sesamin: anti-oxidative activity $(2,3)$; cholesterol and lipid-lowering (4-8); protection against liver damage $(8-10)$; synergy with $\alpha$-tocopherol $(6,11)$ and improvement in the bioavailability of $\gamma$-tocopherol (12-14); anticarcinogenic activities $(15,16)$; and precursors of mammalian lignans $(17,18)$. It has been also reported that sesamin suppressed the development of hypertension in rats $(2,19-26)$.

There are also clinical studies about the effects of ses-

E-mail: t.miyawaki@mhc.west.ntt.co.jp amin in humans. Hirata et al. (5) showed that, in males with hypercholesterolemia, oral administration of 9 capsules (each capsule contained $3.6 \mathrm{mg}$ sesamin and $18 \mathrm{mg}$ vitamin E) per day for $4 \mathrm{wk}$, followed by 18 capsules per day for $4 \mathrm{wk}$ significantly reduced serum total and LDL cholesterol levels. Kiso (8) reported that $36 \mathrm{mg}$ sesamin $2 \mathrm{~h}$ before high intensity exercise suppressed the rise in plasma lipid peroxide levels significantly in 7 male college students. Moritani et al. (27) demonstrated that oral intake of sesamin before smoking in male college students reduced the adverse effects of smoking on the cardiac autonomic nervous system.

However, to our knowledge, there are no data showing the effects of sesamin on blood pressure in humans. In this study, using a double-blind, cross-over, placebocontrolled method, we investigated the effect of 4 -wk administration of sesamin on blood pressure in mildly hypertensive humans.

\section{Methods}

Subjects. Twenty-five middle-aged subjects with mild hypertension (23 men and 2 women, age $49.1 \pm$ $1.8 \mathrm{y}$, body mass index (BMI) $24.6 \pm 0.4 \mathrm{~kg} / \mathrm{m}^{2}$, systolic pressure $137.3 \pm 1.9 \mathrm{mmHg}$, diastolic pressure $87.3 \pm$ $1.2 \mathrm{mmHg}$ ) were recruited from the general population 


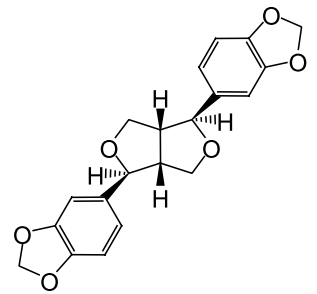

Sesamin

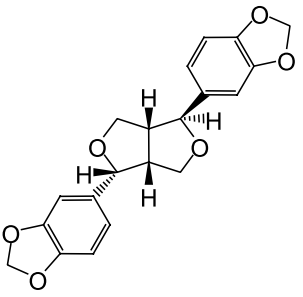

Episesamin
Fig. 1. Chemical structures of sesamin and episesamin.

Table 1. Characteristics of the subjects at baseline.

\begin{tabular}{lrrc}
\hline & Group A & Group B & $p$ value \\
\hline$n$ & 12 & 13 & \\
Age & $51.0 \pm 8.2$ & $47.3 \pm 9.5$ & 0.31 \\
BMI $\left(\mathrm{kg} / \mathrm{m}^{2}\right)$ & $24.1 \pm 0.5$ & $25.0 \pm 0.5$ & 0.24 \\
Systolic BP $(\mathrm{mmHg})$ & $137.3 \pm 3.0$ & $137.4 \pm 2.5$ & 0.98 \\
Diastolic BP $(\mathrm{mmHg})$ & $87.8 \pm 1.7$ & $86.8 \pm 1.7$ & 0.68
\end{tabular}

Data are the means \pm SE. Group A received sesamin first followed by a placebo, and Group B received the placebo first followed by sesamin. BMI, body mass index; BP, blood pressure.

undergoing a yearly health screening or participating in a specific health check-up program performed at NTT West Kyoto Hospital. The subjects had no diseases except mild hypertension, no secondary cause of hypertension, and no medication or dietary supplements that affected blood pressure. Before entering the study, the subjects were told about the nature of the study and written consent was obtained. This study was approved by the ethics committee of NTT West Kyoto Hospital and was performed in accordance with the Helsinki Declaration of 1975 (revised in 1983).

Study design. A double-blind, placebo-controlled cross-over study was undertaken. The subjects were divided into two groups, matched by age, BMI and systolic and diastolic blood pressures (BP). Clinical characteristics of each group of subjects at baseline are given in Table 1. There were no significant changes in age, BMI or systolic and diastolic BP between the groups.

For administration period 1 (September to October), 12 subjects (Group A) were allocated to 4-wk intake of capsules with sesamin and 13 subjects (Group B) to 4wk intake of a placebo. After a 4-wk wash-out period, the subjects received the alternative administration for an additional 4 wk (November to December) as administration period 2. The subjects reported to the hospital five times to have the BP and body weight measured, before and after administration periods 1 and 2, and subsequently $4 \mathrm{wk}$ after the end of administration period 2.

Sesamin capsules were prepared as a mixture of sesamin and episesamin at about a $1: 1$ molar ratio (purity $97.2 \%$, and the remainder was other sesame contents, such as other lignans and sterols) (LOT No. 020904), provided by Suntory Ltd. (Osaka, Japan). Each sesamin capsule contained $10 \mathrm{mg}$ sesamin and $180 \mathrm{mg}$ wheat germ oil. The placebo capsule contained only $180 \mathrm{mg}$ wheat germ oil. Capsules containing sesamin and the placebo were the same shape and color, and both subjects and investigators were blinded as to the composition of the capsules. Group A and Group B subjects took 3 capsules twice per day with water after breakfast and dinner during the administration periods. The subjects were instructed not to change their diets (including meal times and salt intake) or exercise routines throughout this study.

Measurement of body weight and blood pressure. After an overnight fast, body weight and blood pressure were measured. BP was recorded at 10 a.m. after 30 minutes' rest, using a mercury sphygmomanometer (BP203RVIII, Japan Colin Limited, Tokyo, Japan) on the right upper arm in the sitting position. We recorded BP several times and adopted the average of three measurements after almost stable BP.

Statistical analysis. Data are expressed as the means \pm SE. The unpaired $t$-test was used to compare subjects in Group A and B in terms of baseline characteristics. The paired $t$-test was used to compare BP before and after the administration periods. Repeated analyses of covariance were used to compare the BMIs and changes in BP. $p<0.05$ was considered significant. The statistical software StatView 5.0 (SAS Institute Inc., $\mathrm{NC}$, USA) was used for analyses.

\section{Results}

Throughout the study, no significant complaints or side effects were induced by sesamin. The average BMI of the subjects did not change significantly from the beginning of period 1 to the end of period 2 in either Group A or B ( $p=0.13,0.29$, respectively). Figure 2 shows the changes in the average BP levels throughout this study. In Group A (solid line), systolic and diastolic BP after administration of sesamin were significantly decreased compared with before administration in period 1 ( $p=0.04,0.04$, respectively). After the 4-wk washout, systolic and diastolic BP were slightly increased and after administration of the placebo in the period 2, BP was also increased. Meanwhile, in Group B (broken line), systolic and diastolic BP after administration of the placebo was slightly decreased in period 1 , but this was not significant $(p=0.49,0.74$, respectively). After the washout, BP was slightly increased, but subsequently decreased after period 2 , but not significantly ( $p=0.43,0.30$, respectively). After the 4 -wk washout from period 2, BP increased in both groups. Figure 3 shows the changes in BP in the sesamin and placebo groups throughout the study. The mean decreased levels of BP following the administration of sesamin were $3.5 \mathrm{mmHg}$ systolic $\mathrm{BP}$ and $1.9 \mathrm{mmHg}$ diastolic BP (systolic: 137.6 \pm 2.2 to $134.1 \pm 1.7 \mathrm{mmHg}$, $p=0.044$, diastolic: $87.7 \pm 1.3$ to $85.8 \pm 1.0 \mathrm{mmHg}$, $p=0.045)$; however, they were little changed by the placebo (systolic: $135.0 \pm 1.8$ to $135.1 \pm 1.7 \mathrm{mmHg}, p=$ 0.92 , diastolic: $85.9 \pm 1.2$ to $86.6 \pm 1.2 \mathrm{mmHg}, p=$ 

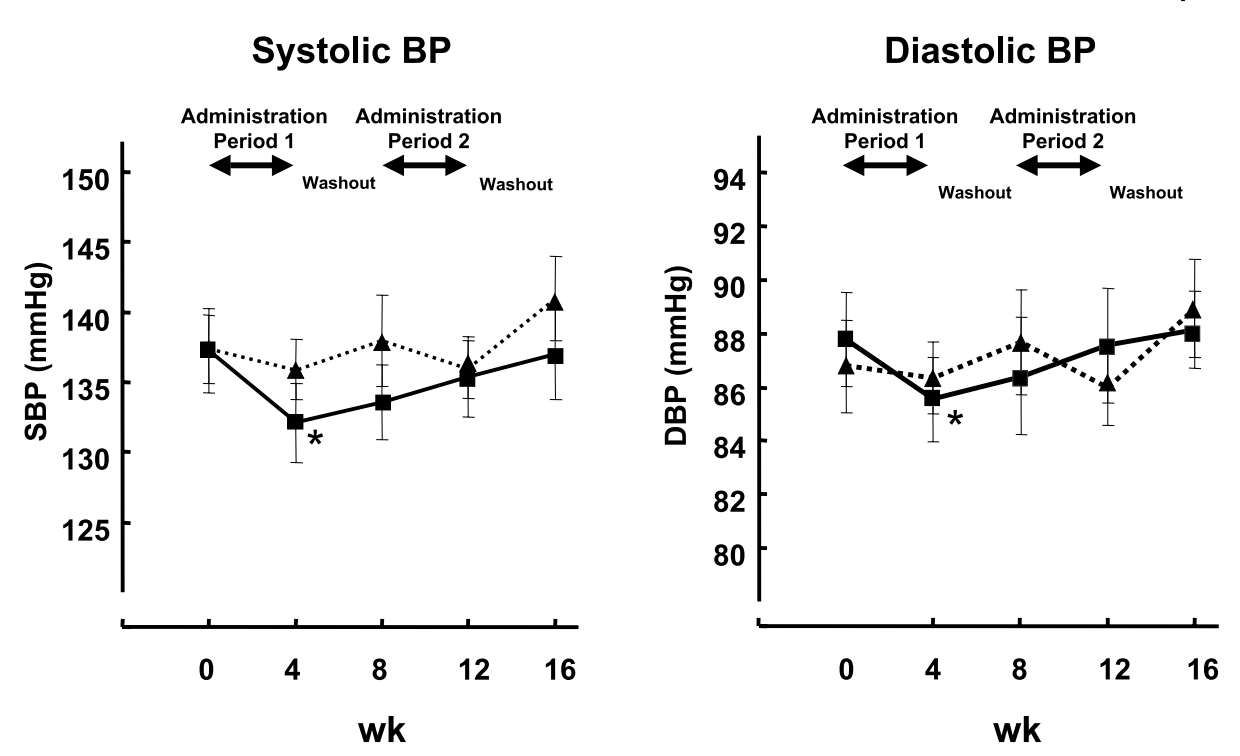

Fig. 2. Changes in the average levels of BP in Groups A and B. Group A (solid line) represents subjects who received sesamin first followed by a placebo, and Group B (broken line) represents subjects who received the placebo first followed by sesamin. ${ }^{*} p<0.05$ compared with BP before the administration period.

\section{Systolic BP}

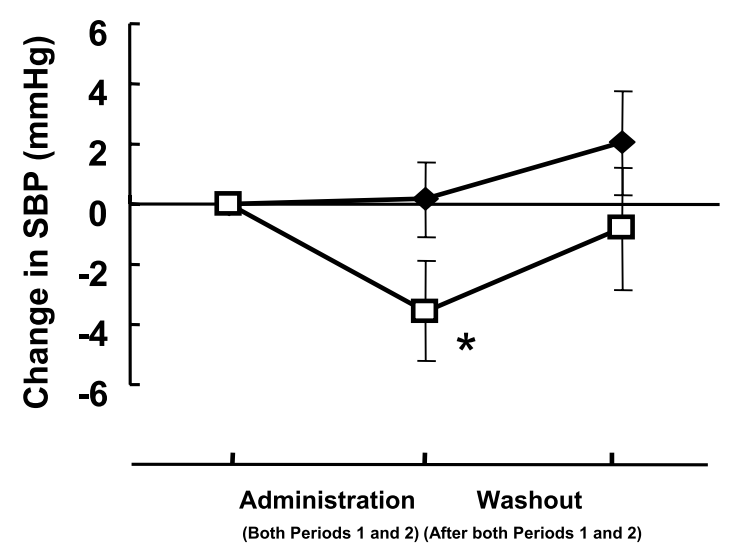

\section{Diastolic BP}

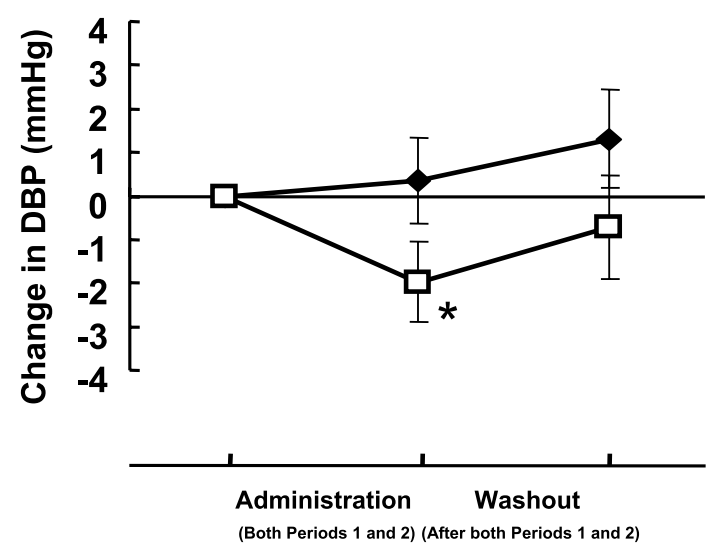

Fig. 3. Changes in the average levels of BP with sesamin and the placebo during the study (Data for "Administration period" includes those in both Periods A and B. Data for "Washout" includes those after both Periods A and B). ${ }^{*} p<0.05$ compared with BP before the administration period.

0.48), and the collective data showed that BP was significantly decreased by the administration of sesamin. After the 4-wk washout from the end of period 2, the average $\mathrm{BP}$ in the sesamin group increased to almost the baseline BP.

\section{Discussion}

In this study, we explored the possible hypotensive effect of sesamin in humans. Our study revealed that 4wk administration of $60 \mathrm{mg}$ sesamin caused a decrease in BP with an average of $3.5 \mathrm{mmHg}$ for systolic BP and $1.9 \mathrm{mmHg}$ for diastolic BP. To our knowledge, this is the first study to investigate the antihypertensive effect of sesamin in humans.

Epidemiological studies suggested that a slight decrease in BP reduces the rate of cardiovascular diseases. The INTERSALT study reported that a 2$3 \mathrm{mmHg}$ decrease in systolic BP is associated with a $4 \%$ lower risk of coronary death and a $6 \%$ lower risk of stroke death in middle age in the US and UK (28). It was also reported that a $2 \mathrm{mmHg}$ decrease in systolic BP is associated with a $6.4 \%$ lower risk of mortality due to cerebral vessel disease in Japan (29); therefore, it is considered that the BP reduction achieved by sesamin in 
this study may be meaningful to prevent cardiovascular diseases.

In period 2, BP was decreased in Group B (sesamin administration) but not significantly, compared with the significant decrease in Group A in period 1. Considering the increased BP levels of Group A at the end of period 2 (placebo) in winter (November to December), the relative cardiovascular load in winter could elevate $\mathrm{BP}$ and cause a bias against the hypotensive effect of sesamin.

Previous studies reported the biophysical mechanism of the antihypertensive effect of sesamin and its metabolites in rats. Sesamin ameliorated the development of deoxycorticosterone acetate (DOCA)-salt-induced vascular hypertrophy and prevented the development of hypertension $(19,24)$, as well as the development of hypertension and cardiac hypertrophy in two-kidney, one-clip hypertensive rats (20), and delayed the development of hypertension and ameliorated both vascular hypertrophy and renal damage in salt-loaded strokeprone spontaneously hypertensive rats (21). Accumulating evidence indicates that oxidative stress, especially increased $\mathrm{O}_{2}{ }^{-}$production, is closely related to the development of hypertension (30) and it is suggested that the mechanism of the antihypertensive effects of sesamin is its antioxidative activities and, in part, sesamin improved impaired endothelium-dependent vasodilatory responses and vasorelaxation $(23,24,31)$. It is also thought that sesamin may improve hypertension by its ability to induce nitric oxide and inhibit endothelin-1 production from human umbilical vein endothelial cells (25). Furthermore, it is suggested that the metabolic products had potent radical-scavenging activities in vitro (2) and that the enhancement of endotheliumdependent vasorelaxation induced by sesamin metabolites is one of the important mechanisms of the in vivo antihypertensive effect of sesamin (31). Taken together, it is considered that sesamin might involve several pathways in its hypotensive effects (26).

We used sesamin as a mixture of sesamin and episesamin. It has been noted that, compared with sesamin, episesamin is more competent in increasing the activity and gene expression of hepatic fatty acid oxidation enzymes of rats (32). Our colleagues showed that episesamin metabolites, as well as sesamin metabolites, could induce vasorelaxation and improve vasoconstrictor responses in vitro (unpublished data); therefore, both sesamin and episesamin may also contribute to the antihypertensive activity to some degree. In this study, furthermore, the subjects included both men and women. A recent study demonstrated sex differences in the inhibition of gamma-tocopherol metabolism by dietary sesame oil (14). Further investigations are needed to explore the biophysical mechanisms of sesamin, including the comparative effects of sesamin and episesamin, and sex differences in the effects of sesamin in humans.

The present study showed that the degree of BP reduction by sesamin was not so large, and there was little difference in BP reduction in terms of the age and body weight of the subjects. This may have been partly due to the small number of subjects and low-dose administration of sesamin compared to that in rats per $\mathrm{kg}$ of body weight. The amount of sesamin administered (60 mg per day) was decided according to a previous clinical study in which the hypocholesterolemic effect of sesame lignan and sesamin induced almost no side effects in any subjects (5). Sixty milligrams of sesamin is equivalent to about 8 tablespoons of sesame, and it is difficult to get this amount from sesame seeds everyday. If this amount of sesame seeds is eaten continuously, there is a possibility of eating too much oil; therefore, any alternative method of taking sesamin, such as the capsules containing purified sesamin in this study, may be needed. Furthermore, the optimum amount of sesamin, administration interval, and administration times needed to decrease BP should also be further investigated.

In conclusion, our study showed that 4-wk administration of sesamin significantly decreased both systolic and diastolic BP in mildly hypertensive humans. These results suggest that sesamin has antihypertensive effects in humans.

\section{REFERENCES}

1) Fukuda Y, Nagata M, Osawa T, Namiki M. 1986. Contribution of lignan analogues to antioxidative activity of refined unroasted sesame seed oil. J Am Oil Chem Soc 63: 1027-1031.

2) Nakai M, Harada M, Nakahara K, Akimoto K, Shibata H, Miki W, Kiso Y. 2003. Novel antioxidative metabolites in rat liver with ingested sesamin. J Agric Food Chem 51: 1666-1670.

3) Ikeda T, Nishijima Y, Shibata H, Kiso Y, Ohnuki K, Fushiki T, Moritani T. 2003. Protective effect of sesamin administration on exercise induced lipid peroxidation. Int J Sports Med 24: 530-534.

4) Hirose N, Inoue T, Nishihara K, Sugano M, Akimoto K, Shimizu S, Yamada H. 1991. Inhibition of cholesterol absorption and synthesis in rats by sesamin. J Lipid Res 32: 629-638.

5) Hirata F, Fujita K, Ishikura Y, Hosoda K, Ishikawa T, Nakamura H. 1996. Hypocholesterolemic effect of sesame lignan in humans. Atherosclerosis 122: 135-136.

6) Nakabayashi A, Kitagawa Y, Suwa Y, Akimoto K, Asami S, Shimizu S, Hirose N, Sugano M, Yamada H. 1995. alpha-Tocopherol enhances the hypocholesterolemic action of sesamin in rats. Int J Vitam Nutr Res 65: 162168.

7) Ogawa H, Sasagawa S, Murakami T, Yoshizumi H. 1995. Sesame lignans modulate cholesterol metabolism in the stroke-prone spontaneously hypertensive rat. Clin Exp Pharmacol Physiol 22: S310-312.

8) Kiso Y. 2004. Antioxidative roles of sesamin, a functional lignan in sesame seed, and its effect on lipid-and alcohol-metabolism in the liver: A DNA microarray study. Biofactors 21: 191-196.

9) Akimoto K, Kitabawa Y, Akamatsu T, Hirose N, Sugano M, Shimizu S, Yamada H. 1993. Protective effects of sesamin against liver damage caused by alcohol or carbon tetrachloride in rodents. Ann Nutr Metab 37: 218-224.

10) Kiso Y, Tsuruoka N, Kidokoro A, Matsumoto I, Abe K. 2005. Sesamin ingestion regulates the transcription 
levels of hepatic metabolizing enzymes for alcohol and lipids in rats. Alcohol Clin Exp Res 29: 116S-120S.

11) Yamashita K, Kagaya M, Higuchi N, Kiso Y. 2000. Sesamin and alfa-tocopherol synergistically suppress lipidperoxide in rats fed with a high docosahexaenoic acid diet. Biofactors 11: 11-13.

12) Kamal-Eldin A, Pettersson D, Appelqvist LA. 1995. Sesamin (a compound from sesame oil) increases tocopherol levels in rats fed ad libitum. Lipids 30: 499-505.

13) Frank J, Kamal-Eldin A, Traber MG. 2004. Consumption of sesame oil muffins decreases the urinary excretion of gamma-tocopherol metabolities in humans. Ann NY Acad Sci 1031: 365-367.

14) Frank J, Lee S, Leonard SW, Atkinson JK, Kamal-Eldin A, Traber MG. 2008. Sex differences in the inhibition of gamma-tocopherol metabolism by a single dose of dietary sesame oil in healthy subjects. Am J Clin Nutr 87: 1723-1729.

15) Hirose N, Doi F, Ueki T, Akazawa K, Chijiiwa K, Sugano M, Akimoto K, Shimizu S, Yamada H. 1992. Suppressive effect of sesamin against 7,12-dimethylbenz[a]-anthracene induced rat mammary carcinogenesis. Anticancer Res 12: 1259-1265.

16) Miyahara $Y$, Komiya T, Katsuzaki H, Imai K, Nakagawa M, Ishii Y, Hibasami H. 2000. Sesamin and episesamin induce apoptosis in human lymphoid leukemia. Int $J$ Mol Med 6: 43-46.

17) Peñalvo JL, Heinonen SM, Aura AM, Adlercreutz H. 2005. Dietary sesamin is converted to enterolactone in humans. J Nutr 135: 1056-1062.

18) Liu Z, Saarinen NM, Thompson LU. 2006. Sesamin is one of the major precursors of mammalian lignans in sesame seed (Sesamum indicum) as observed in vitro and in rats. J Nutr 136: 906-912.

19) Matsumura $Y$, Kita $S$, Morimoto S, Akimoto K, Furuya M, Oka N, Tanaka T. 1995. Antihypertensive effect of sesamin. I. Protection against deoxycorticosterone acetate-salt-induced hypertension and cardiovascular hypertrophy. Biol Pharm Bull 18: 1016-1019.

20) Kita S, Matsumura Y, Morimoto S, Akimoto K, Furuya M, Oka N, Tanaka T. 1995. Antihypertensive effect of sesamin. II. Protection against two-kidney, one-clip renal hypertension and cardiovascular hypertrophy. Biol Pharm Bull 18: 1283-1285.

21) Matsumura Y, Kita S, Tanida Y, Taguchi Y, Morimoto S, Akimoto K, Tanaka T. 1998. Antihypertensive effect of sesamin. III. Protection against development and maintenance of hypertension in stroke-prone spontaneously hypertensive rats. Biol Pharm Bull 21: 469-473.

22) Noguchi T, Ikeda K, Sasaki Y, Yamamoto J, Seki J, Yama- gata K, Nara Y, Hara H, Kakuta H, Yamori Y. 2001. Effects of vitamin $\mathrm{E}$ and sesamin on hypertension and cerebral thrombogensis in stroke-prone spontaneously hypertensive rats. Hypertens Res 24: 735-742.

23) Nakano D, Itoh C, Takaoka M, Kiso Y, Tanaka T, Matsumura Y. 2002. Antihypertensive effect of sesamin. IV. Inhibition of vascular superoxide production by sesamin. Biol Pharm Bull 25: 1247-1249.

24) Nakano D, Itoh C, Ishii F, Kawanishi H, Takaoka M, Kiso Y, Tsuruoka N, Tanaka T, Matsumura Y. 2003. Effects of sesamin on aortic oxidative stress and endothelial dysfunction in deoxycorticosterone acetate-salt hypertensive rats. Biol Pharm Bull 26: 1701-1705.

25) Lee CC, Chen PR, Lin S, Tsai SC, Wang BW, Chen WW, Tsai CE, Shyu KG. 2004. Sesamin induces nitric oxide and decreases endothelin-1 production in HUVECs: possible implications for its antihypertensive effect. J Hypertens 22: 2329-2338.

26) Nakano D, Takaoka M, Kiso Y, Matsumura Y. 2004. Antihypertensive effect of sesamin. Vasc Dis Prev 1: 233-241.

27) Moritani T, Hamada T, Kimura T, Hayashi T, Kiso Y. 2003. Changes in cardiac autonomic activities and antioxidant effects of sesamin during smoking. Med Sci Sports Exerc 35: S269.

28) Stamler J, Rose G, Stamler R, Elliott P, Dyer A, Marmot M. 1989. INTERSALT study findings. Public health and medical care implications. Hypertension 14: 570-577.

29) Japanese Society of Hypertension. 2006. Epidemiology of hypertension. Japanese society of hypertension guidelines for the management of hypertension (JSH 2004). Hypertens Res 29 (Suppl): S1-S6.

30) Zalba G, Josè GS, Moreno MU, Fortuño MA, Fortuño A, Beaumont FJ, Díez J. 2001. Oxidative stress in arterial hypertension. Role of $\mathrm{NAD}(\mathrm{P}) \mathrm{H}$ oxidase. Hypertension 38: 1395-1399.

31) Nakano D, Kwak CJ, Fujii K, Ikemura K, Satake A, Ohkita M, Takaoka M, Ono Y, Nakai M, Tomimori N, Kiso Y, Matsumura Y. 2006. Sesamin metabolites induce an endothelial nitric oxide-dependent vasorelaxation through their antioxidative property-independent mechanisms: Possible involvement of the metabolites in the antihypertensive effect of sesamin. J Pharm Exp Ther 318: 328-335.

32) Kushiro M, Masaoka T, Hageshita S, Takahashi Y, Ide T, Sugano M. 2002. Comparative effect of sesamin and episesamin on the activity and gene expression of enzymes in fatty acid oxidation and synthesis in rat liver. J Nutr Biochem 13: 289-295. 